\title{
Convergence rates of nonlinear Stokes problems in homogenization
}

Juan Wang ${ }^{1 *}$ and Jie Zhao'

"Correspondence:

wangjuan03022204@163.com

${ }^{1}$ College of Science, Zhongyuan University of Technology,

Zhengzhou, China

\begin{abstract}
In this paper, we study the convergence rates of solutions in homogenization of nonlinear Stokes Dirichlet problems. The main difficulty of this work is twofold. On the one hand, the nonlinear Stokes problems do not fit the standard framework of second-order elliptic equations in divergence form. On the other hand, nonlinear problems may cause new difficulties in the estimation of the quantity as well as first-order approximate term. As a consequence, we establish the sharp rates of convergence in $H^{1}$ and $L^{2}$. This work may be regarded as an extension of the approach for the linear Stokes problems to the nonlinear case.
\end{abstract}

MSC: Primary 35J15; secondary 35J25

Keywords: Homogenization; Convergence rates; Stokes problems; Smoothing operators

\section{Introduction}

The main purpose of this paper is to establish the sharp rates of convergence in $H^{1}$ and $L^{2}$ for nonlinear Stokes problems with the Dirichlet boundary condition. More precisely, let $\Omega$ be a bounded $C^{1,1}$ domain in $\mathbb{R}^{n}, n \geq 3$. Let $u_{\varepsilon} \in H^{1}\left(\Omega ; \mathbb{R}^{n}\right)$ be a weak solution to the following problem, which arose in fluid dynamics with porous media:

$$
\begin{cases}L_{\varepsilon} u_{\varepsilon}+\nabla p_{\varepsilon}=F & \text { in } \Omega, \\ \operatorname{div} u_{\varepsilon}=f & \text { in } \Omega, \\ u_{\varepsilon}=g & \text { on } \partial \Omega\end{cases}
$$

with the compatibility condition

$$
\int_{\Omega} f d x=\int_{\partial \Omega} g \cdot n d \sigma
$$

where $n$ is the outward unit normal to $\partial \Omega$.

Throughout this paper, the summation convention is used. The nonlinear operator $L_{\varepsilon}$ is defined by

$$
L_{\varepsilon}=-\operatorname{div} A(x / \varepsilon, \nabla)
$$

(c) The Author(s) 2019. This article is distributed under the terms of the Creative Commons Attribution 4.0 International License (http://creativecommons.org/licenses/by/4.0/), which permits unrestricted use, distribution, and reproduction in any medium, provided you give appropriate credit to the original author(s) and the source, provide a link to the Creative Commons license, and indicate if changes were made. 
We will assume that the function $A$ satisfies the periodicity condition

$$
A(y+Y, \xi)=A(y, \xi) \quad \text { for } Y=[0,1)^{n} \simeq \mathbb{R}^{n} / \mathbb{Z}^{n}
$$

coerciveness and growth conditions

$$
\begin{aligned}
& \left\langle A(y, \xi)-A\left(y, \xi^{\prime}\right), \xi-\xi^{\prime}\left|\geq \lambda_{1}\right| \xi-\left.\xi^{\prime}\right|^{2},\right. \\
& \left|A(y, \xi)-A\left(y, \xi^{\prime}\right)\right| \leq \frac{1}{\lambda_{1}}\left|\xi-\xi^{\prime}\right|,
\end{aligned}
$$

for all $y \in \mathbb{R}^{n}$ and $\xi, \xi^{\prime} \in \mathbb{R}^{n}$, where $\lambda_{1}>0$. We impose the smoothness condition

$$
\left|A(y, \xi)-A\left(y^{\prime}, \xi\right)\right| \leq \lambda_{2}\left|y-y^{\prime}\right|^{\alpha}, \quad F \in H^{-1}(\Omega), f \in L^{2}(\Omega), g \in H^{1 / 2}(\partial \Omega)
$$

where $\lambda_{2}>0$ and $0<\alpha \leq 1$. Without loss of generality, we also assume that

$$
\frac{1}{|\Omega|} \int_{\Omega} p_{\varepsilon} d x=0
$$

Associated with (1.1) is the homogenized problem

$$
\begin{cases}L_{0} u_{0}+\nabla p_{0}=F & \text { in } \Omega, \\ \operatorname{div} u_{0}=f & \text { in } \Omega, \\ u_{0}=g & \text { on } \partial \Omega .\end{cases}
$$

The homogenized operator is defined by

$$
L_{0}=-\operatorname{div} Q(\nabla) \text {, }
$$

where the function $Q$ is given, for each $\xi \in \mathbb{R}^{n}$, by

$$
Q(\xi)=\int_{Y} A\left(y, \xi+\nabla_{y} N(y, \xi)\right) d y .
$$

The periodic functions $(N, \chi) \in H^{1}\left(\mathbb{R}^{n}\right) \times L^{2}\left(\mathbb{R}^{n}\right)$ are the so-called correctors, satisfying the following cell problem:

$$
\left\{\begin{array}{l}
\operatorname{div} A\left(y, \xi+\nabla_{y} N(y, \xi)\right)-\nabla \chi=0 \quad \text { in } Y \\
N(y+Y, \xi)=N(y, \xi) \\
\int_{Y} N(y, \xi) d y=0 .
\end{array}\right.
$$

It is well known that, by the homogenization theory of Stokes problems, the solution $u_{\varepsilon} \rightarrow u_{0}$ weakly in $H^{1}\left(\Omega ; \mathbb{R}^{n}\right), p_{\varepsilon} \rightarrow p_{0}$ weakly in $L^{2}(\Omega)$, and $A\left(x / \varepsilon, \nabla u_{\varepsilon}\right) \rightarrow Q\left(\nabla u_{0}\right)$ weakly in $L^{2}\left(\Omega, \mathbb{R}^{n \times n}\right)$, as $\varepsilon \rightarrow 0$. The existence and convergence results of the weak solution to problem (1.1) may be found in $[4,12]$.

The convergence rate estimate is one of the fundamental issues in quantitative homogenization. There are many such classic works about convergence results of solutions in homogenization of second-order elliptic equations with the various settings. In 2011, Gérard 
and Masmoudi [6] got the $L^{2}$ convergence for the Neumann boundary layer problems. In 2012, Kenig, Lin, and Shen [13] established $L^{2}$ as well as $H^{\frac{1}{2}}$ convergence in Lipschitz domains for Dirichlet and Neumann problems. In 2013, Aleksanyan, Shahgholian, and Sjölin [1,2] proved pointwise and $L^{p}$ convergence estimates for fixed operators and oscillating Dirichlet boundary data. In 2014, Kenig, Lin, and Shen [14] also obtained $W^{k, p}$ convergence rates of Dirichlet or Neumann problems for the second-order equations with rapidly oscillating periodic coefficients by using the asymptotic estimates of the Green or Neumann functions. In 2015, the second author [24] proved the pointwise as well as $W^{1, p}$ convergence estimates for the fixed operators and oscillating Neumann boundary data by utilizing oscillation integral estimates in Fourier analysis. In 2016, Shen [17] proved the $L^{q}$ convergence rates with Dirichlet or Neumann problems with no smoothness assumption on the coefficients. In 2018, Shen and Zhuge [19] got the $L^{2}$ convergence rate for the Neumann problems with first-order oscillating Neumann boundary data.

For the case of Stokes problems, some outstanding results about regularity and convergence of solutions in homogenization were established by $\mathrm{Gu}$ and Shen in a series of papers. The uniform interior estimates and boundary Hölder estimates for the Dirichlet problem have been established in [8]. Then, the authors in [9] obtained the sharp boundary regularity estimates in homogenization of Dirichlet problem. In 2015, Gu [7] also proved convergence rates in $L^{2}$ and $H^{1}$ of Dirichlet problems for linear Stokes systems. In 2017, $\mathrm{Gu}$ and Shen [10] got the asymptotic behaviors of the Green functions as well as the convergence rates in $L^{p}$ and $L^{\infty}$ for solutions. Recently, other authors have also been interested in the regularity estimates for the Stokes problems, see $[3,5,11,23]$ and their references for more results.

The main difficulty of this work is twofold. On the one hand, the nonlinear Stokes problems do not fit the standard framework of second-order elliptic equations in divergence form, which is caused by the pressure term. On the other hand, nonlinear problems may cause new difficulties in the estimation of the quantity as well as first-order approximate term.

The motivation for studying this paper is inspired by the technology used to deal with linear Stokes problems studied by Gu in [7]. The novelty of this paper lies in that it may be regarded as an extension of the approach for the linear Stokes problems to the nonlinear case. As the author knows, very few convergence rate results are known in the field of nonlinear Stokes problems.

The following are the main results of this paper.

Theorem 1 Let $\Omega$ be a bounded $C^{1,1}$ domain in $\mathbb{R}^{n}$. Let $u_{\varepsilon} \in H^{1}\left(\Omega ; \mathbb{R}^{n}\right)$ and $u_{0} \in$ $H^{2}\left(\Omega ; \mathbb{R}^{n}\right)$ be the weak solutions of the mixed boundary value problems (1.1) and (1.9), respectively. Then, under assumptions (1.2)-(1.8), there exists a constant $C$ such that

$$
\left\|u_{\varepsilon}-u_{0}-\varepsilon N\left(x / \varepsilon, T_{\varepsilon}\left(\nabla \tilde{u}_{0}\right)\right)+\omega_{\varepsilon}\right\|_{H_{0}^{1}(\Omega)} \leq C \varepsilon\left\|u_{0}\right\|_{H^{2}(\Omega)},
$$

where $T_{\varepsilon}$ is the smoothing operator, $\tilde{u}_{0}$ is an extension of $u_{0}$, and $\omega_{\varepsilon}$ is an approximate function.

Theorem 2 Under the same assumptions as Theorem 1, there exists a constant $C$ such that

$$
\left\|u_{\varepsilon}-u_{0}-\varepsilon N\left(x / \varepsilon, T_{\varepsilon}\left(\nabla \tilde{u}_{0}\right)\right)\right\|_{H^{1}(\Omega)} \leq C \varepsilon^{1 / 2}\left\|u_{0}\right\|_{H^{2}(\Omega)} .
$$


Theorem 3 Under the same conditions as Theorem 1, there exists a constant $C$ such that

$$
\left\|u_{\varepsilon}-u_{0}\right\|_{L^{2}(\Omega)} \leq C \varepsilon^{1 / 2}\left\|u_{0}\right\|_{H^{2}(\Omega)} .
$$

The rest of the paper is organized as follows. Section 2 contains some basic definitions and useful propositions which will play important roles in obtaining convergence rates. In Sect. 3, we show that the solution $u_{\varepsilon}$ of nonlinear Stokes problems is convergent to the solution $u_{0}$ of the corresponding homogenized problems, this is based on using of a smoothing operator as well as homogenization tools.

\section{Preliminaries}

We begin by specifying some of our notations.

Let $B_{r}(x)$ denote an open ball with center $x$ and radius $r . \Omega_{\varepsilon}=\left\{x \in \mathbb{R}^{n}: \operatorname{dist}(x, \partial \Omega) \leq \varepsilon\right\}$. Since $\Omega$ is Lipschitz, then there exists a bounded extension operator $E: H^{2}(\Omega) \rightarrow H^{2}\left(\mathbb{R}^{n}\right)$ such that $\tilde{u}_{0}$ is an extension of $u_{0}$ satisfying $\left\|\widetilde{u}_{0}\right\|_{H^{2}\left(\mathbb{R}^{n}\right)} \leq C\left\|u_{0}\right\|_{H^{2}(\Omega)}$. We also set $\varphi \in$ $C_{0}^{\infty}\left(\Omega ; \mathbb{R}^{n}\right)$ is a smooth function and $\|\varphi\|_{H^{1}\left(\mathbb{R}^{n}\right)} \leq C\|\varphi\|_{H^{1}(\Omega)}$. We choose a cut-off function $\eta_{\varepsilon} \in C_{0}^{\infty}\left(\mathbb{R}^{n}\right)$, which satisfies the conditions: $\operatorname{supp}\left(\eta_{\varepsilon}\right) \subset \Omega_{\varepsilon},\left.\eta_{\varepsilon}\right|_{\partial \Omega} \equiv 1$ and $\left|\nabla \eta_{\varepsilon}\right| \leq C / \varepsilon$. In this paper, $C$ always denotes a positive constant which may vary in different formulas.

Associated with operator $L_{\varepsilon}$ in (1.1), the homogenized operator is

$$
L_{0}=-\operatorname{div} Q(\nabla) \quad \text { in } \Omega \text {, }
$$

the function $Q$ and corrector function $N\left(x / \varepsilon, \nabla u_{0}\right)$ are defined in (1.10), (1.11), respectively, and they satisfy the following properties.

Proposition 2.1 The function $Q$ defined in (1.10) satisfies the analogous properties as function A:

$$
\left|Q(\xi)-Q\left(\xi^{\prime}\right)\right| \leq C\left|\xi-\xi^{\prime}\right|
$$

and

$$
\left\langle Q(\xi)-Q\left(\xi^{\prime}\right), \xi-\xi^{\prime}\right\rangle \geq C\left|\xi-\xi^{\prime}\right|^{2}
$$

for all $\xi, \xi^{\prime} \in \mathbb{R}^{n}$.

Proof The proof could be found in [16], which is similar to the linear operator case. Obviously, this shows that the homogenized operator $L_{0}$ still satisfies the same coerciveness and growth conditions.

Proposition 2.2 The function $N(\cdot, \xi) \in H^{1}(Y)$ is a weak solution to (1.11). Then we have

$$
\|N(y, \xi)\|_{L^{\infty}(Y)} \leq C|\xi|, \quad \int_{Y}\left|\nabla_{\xi} N(y, \xi)\right|^{2} d y \leq C
$$

and

$$
\int_{Y}|N(y, \xi)|^{2} d y+\int_{Y}\left|\nabla_{y} N(y, \xi)\right|^{2} d y \leq C|\xi|^{2}
$$

for all $y, \xi \in \mathbb{R}^{n}$. 
Proof These estimates have been proved in [16] and [22]. Multiplying both sides of (1.11) by $N(y, \xi)$ and integrating by parts, one could get the desired results.

The next proposition is the special relation between $Q$ and $A$ in homogenization. We also call them the flux correctors.

\section{Proposition 2.3 Let}

$$
F(y, \xi)=Q(\xi)-A(y, \xi+\nabla N(y, \xi))
$$

where $y \in Y$ and $\xi \in \mathbb{R}^{n}$. Together with (1.10) and (1.11), it is easy to know that $F(\cdot, \xi)$ satisfies conditions $\int_{Y} F(y, \xi) d y=0$ and $\operatorname{div}_{y} F(y, \xi)=\nabla \chi$. Then there exists $\Phi_{i j}(\cdot, \xi) \in H^{1}\left(\mathbb{R}^{n}\right)$ such that

$$
\Phi_{i j}(y, \xi)=-\Phi_{j i}(y, \xi) \quad \text { and } \quad F_{j}(y, \xi)=\frac{\partial \Phi_{i j}(y, \xi)}{\partial y_{i}}+\chi_{j}
$$

Moreover,

$$
\int_{Y}\left|\Phi_{i j}(y, \xi)\right|^{2} d y+\int_{Y}\left|\nabla_{\xi} \Phi_{i j}(y, \xi)\right|^{2} d y \leq C .
$$

Proof The linear operator case is well known (see, for example, [13], Lemma 3.1). This proposition is quite similar to the linear case. Let $f_{j} \in H^{2}(Y)$ be the solution to the cell problem $\triangle f_{j}+\chi_{j}=F_{j}$ in $Y$. Then, we could define $\Phi_{i j}(y, \xi)=\frac{\partial}{\partial y_{i}}\left[f_{j}(y, \xi)\right]-\frac{\partial}{\partial y_{j}}\left[f_{i}(y, \xi)\right]$. From every estimate and (1.11), we may get the desired properties. We refer the reader to [7] for more details.

Recently, the smoothing operators were introduced by Suslina in [20, 21], which was used to establish the convergence estimate in $L^{2}$ for a broad class of elliptic or parabolic operators. This work seems to extend the usage of smoothing operators to the case of nonlinear Stokes problems.

Fix $\psi \in C_{0}^{\infty}\left(B_{1}(0)\right)$ such that $\psi \geq 0$ and $\int_{\mathbb{R}^{n}} \psi d x=1$. Define operator $T_{\varepsilon}$ on $L^{2}$ as

$$
T_{\varepsilon}(u)(x)=u * \psi_{\varepsilon}=\int_{\mathbb{R}^{n}} u(x-y) \psi_{\varepsilon}(y) d y,
$$

where $\psi_{\varepsilon}(x)=\varepsilon^{-n} \psi(x / \varepsilon)$.

Proposition 2.4 If $u_{0} \in H^{2}\left(\mathbb{R}^{n}\right)$, then

$$
\left\|\nabla u_{0}-T_{\varepsilon}\left(\nabla u_{0}\right)\right\|_{L^{2}\left(\mathbb{R}^{n}\right)} \leq C \varepsilon\left\|\nabla^{2} u_{0}\right\|_{L^{2}\left(\mathbb{R}^{n}\right)}
$$

and

$$
\left\|T_{\varepsilon}\left(\nabla^{2} u_{0}\right)\right\|_{L^{2}\left(\mathbb{R}^{n}\right)} \leq C\left\|\nabla^{2} u_{0}\right\|_{L^{2}\left(\mathbb{R}^{n}\right)}
$$

Proof By Parseval's theorem and Hölder's inequality, we could get the desired result. The proof could be found in [17]. 
Proposition 2.5 If $u_{0} \in H^{2}\left(\mathbb{R}^{n}\right)$, then

$$
\left\|T_{\varepsilon}\left(\nabla u_{0}\right)\right\|_{L^{2}\left(\Omega_{\varepsilon}\right)} \leq C \varepsilon^{1 / 2}\left\|u_{0}\right\|_{H^{2}\left(\mathbb{R}^{n}\right)}
$$

Proof This estimate could be proved by Fubini's theorem. See [15] or [18] for the detailed proof.

\section{Proofs of theorems}

The goal of this section is to establish $H^{1}$ and $L^{2}$ convergence rates of solutions.

Proof of Theorem 1 Let $\omega_{\varepsilon} \in H^{1}(\Omega)$ be a weak solution of

$$
\begin{cases}L_{\varepsilon} \omega_{\varepsilon}+\nabla\left(p_{\varepsilon}-p_{0}-\chi\right)=0 & \text { in } \Omega, \\ \operatorname{div} \omega_{\varepsilon}=\varepsilon \operatorname{div} N\left(x / \varepsilon, T_{\varepsilon}\left(\nabla \widetilde{u}_{0}\right)\right) & \text { in } \Omega, \\ \omega_{\varepsilon}=\varepsilon N\left(x / \varepsilon, T_{\varepsilon}\left(\nabla \widetilde{u}_{0}\right)\right) & \text { on } \partial \Omega .\end{cases}
$$

We will use $\omega_{\varepsilon}$ to approximate the difference of pressure term.

Introduce the first-order approximation of $u_{\varepsilon}$ :

$$
v_{\varepsilon}=u_{0}+\varepsilon N\left(x / \varepsilon, T_{\varepsilon}\left(\nabla \widetilde{u}_{0}\right)\right)-\omega_{\varepsilon} .
$$

Note that, for any $\varphi \in C_{0}^{\infty}\left(\Omega ; \mathbb{R}^{n}\right)$,

$$
\int_{\Omega} A\left(x / \varepsilon, \nabla u_{\varepsilon}\right) \cdot \nabla \varphi d x-\int_{\Omega} p_{\varepsilon} \operatorname{div} \varphi d x=\int_{\Omega} Q\left(\nabla u_{0}\right) \cdot \nabla \varphi d x-\int_{\Omega} p_{0} \operatorname{div} \varphi d x
$$

A simple calculation then gives that

$$
\begin{aligned}
\int_{\Omega}[ & \left.A\left(x / \varepsilon, \nabla u_{\varepsilon}\right)-A\left(x / \varepsilon, \nabla v_{\varepsilon}\right)\right] \cdot \nabla \varphi d x+\int_{\Omega}\left(p_{0}-p_{\varepsilon}\right) \operatorname{div} \varphi d x \\
= & \int_{\Omega}\left[Q\left(\nabla u_{0}\right)-Q\left(T_{\varepsilon}\left(\nabla \widetilde{u}_{0}\right)\right)\right] \cdot \nabla \varphi d x \\
& +\int_{\Omega}\left[Q\left(T_{\varepsilon}\left(\nabla \widetilde{u}_{0}\right)\right)-A\left(x / \varepsilon, T_{\varepsilon}\left(\nabla \widetilde{u}_{0}\right)+\nabla N\left(x / \varepsilon, T_{\varepsilon}\left(\nabla \widetilde{u}_{0}\right)\right)\right)\right] \cdot \nabla \varphi d x \\
& +\int_{\Omega}\left[A\left(x / \varepsilon, T_{\varepsilon}\left(\nabla \widetilde{u}_{0}\right)+\nabla N\left(x / \varepsilon, T_{\varepsilon}\left(\nabla \widetilde{u}_{0}\right)\right)\right)-A\left(x / \varepsilon, \nabla v_{\varepsilon}\right)\right] \cdot \nabla \varphi d x \\
\doteq & I_{1}+I_{2}+I_{3} .
\end{aligned}
$$

To estimate $I_{1}$, we note that by Proposition 2.1 and Proposition 2.4,

$$
\begin{aligned}
\left|I_{1}\right| & \leq C \int_{\Omega}\left|Q\left(\nabla u_{0}\right)-Q\left(T_{\varepsilon}\left(\nabla \tilde{u}_{0}\right)\right)\right| \cdot|\nabla \varphi| d x \\
& \leq C\left\|\nabla \widetilde{u}_{0}-T_{\varepsilon}\left(\nabla \widetilde{u}_{0}\right)\right\|_{L^{2}\left(\mathbb{R}^{n}\right)}\|\nabla \varphi\|_{L^{2}(\Omega)} \\
& \leq C \varepsilon\left\|u_{0}\right\|_{H^{2}(\Omega)}\|\nabla \varphi\|_{L^{2}(\Omega)} .
\end{aligned}
$$


Next, we shall estimate $I_{2}$. Let

$$
F\left(x / \varepsilon, T_{\varepsilon}\left(\nabla \tilde{u}_{0}\right)\right)=Q\left(T_{\varepsilon}\left(\nabla \tilde{u}_{0}\right)\right)-A\left(x / \varepsilon, T_{\varepsilon}\left(\nabla \tilde{u}_{0}\right)+\nabla N\left(x / \varepsilon, T_{\varepsilon}\left(\nabla \tilde{u}_{0}\right)\right)\right) .
$$

Note that $F\left(x / \varepsilon, T_{\varepsilon}\left(\nabla \tilde{u}_{0}\right)\right)$ is a periodic function with respect to the first variable, and it satisfies the conditions of Proposition 2.3. Then there exists $\Phi_{i j}(\cdot, \xi) \in H^{1}\left(\mathbb{R}^{n}\right)$ satisfying

$$
Q_{j}\left(T_{\varepsilon}\left(\nabla \tilde{u}_{0}\right)\right)-A_{j}\left(y, T_{\varepsilon}\left(\nabla \tilde{u}_{0}\right)+\nabla N\left(y, T_{\varepsilon}\left(\nabla \tilde{u}_{0}\right)\right)\right)=\frac{\partial \Phi_{i j}\left(y, T_{\varepsilon}\left(\nabla \tilde{u}_{0}\right)\right)}{\partial y_{i}}+\chi_{j}
$$

Thus, it gives that

$$
\begin{aligned}
I_{2} & =\int_{\Omega} F\left(x / \varepsilon, T_{\varepsilon}\left(\nabla \tilde{u}_{0}\right)\right) \cdot \nabla \varphi d x \\
& =\int_{\Omega}\left[\frac{\partial \Phi_{i j}\left(y, T_{\varepsilon}\left(\nabla \tilde{u}_{0}\right)\right)}{\partial y_{i}}+\chi_{j}\right] \frac{\partial \varphi}{\partial x_{j}} d x \\
& \doteq I_{21}+I_{22} .
\end{aligned}
$$

For the first term,

$$
\begin{aligned}
I_{21} & =\int_{\Omega} \frac{\partial}{\partial x_{i}}\left(\varepsilon \Phi_{i j}\left(x / \varepsilon, T_{\varepsilon}\left(\nabla \widetilde{u}_{0}\right)\right)\right) \cdot \frac{\partial \varphi}{\partial x_{j}} d x-\int_{\Omega} \varepsilon \frac{\partial \Phi_{i j}(x / \varepsilon, \xi)}{\partial \xi_{h}} \frac{\partial \xi_{h}}{\partial x_{i}} \frac{\partial \varphi}{\partial x_{j}} d x \\
& =-\int_{\Omega} \varepsilon \frac{\partial \Phi_{i j}(x / \varepsilon, \xi)}{\partial \xi_{h}} \frac{\partial \xi_{h}}{\partial x_{i}} \frac{\partial \varphi}{\partial x_{j}} d x
\end{aligned}
$$

where the first term vanishes in the last equality, which depends on the antisymmetry of $\Phi_{i j}$.

As a result, using Proposition 2.3 and Proposition 2.4, we get that

$$
\begin{aligned}
\left|I_{21}\right| & \leq C \varepsilon\left\|T_{\varepsilon}\left(\nabla^{2} \widetilde{u}_{0}\right)\right\|_{L^{2}\left(\mathbb{R}^{n}\right)}\|\nabla \varphi\|_{L^{2}(\Omega)} \\
& \leq C \varepsilon\left\|u_{0}\right\|_{H^{2}(\Omega)}\|\nabla \varphi\|_{L^{2}(\Omega)} .
\end{aligned}
$$

For $I_{3}$, it follows from the growth condition of (1.4) as well as Proposition 2.4 that

$$
\begin{aligned}
\left|I_{3}\right| & \leq C\left(\left\|\nabla \tilde{u}_{0}-T_{\varepsilon}\left(\nabla \widetilde{u}_{0}\right)\right\|_{L^{2}\left(\mathbb{R}^{n}\right)}+\varepsilon\left\|\nabla_{\xi} N(y, \xi)\right\|_{L^{2}(\Omega)}\left\|T_{\varepsilon}\left(\nabla^{2} \widetilde{u}_{0}\right)\right\|_{L^{2}\left(\mathbb{R}^{n}\right)}\right)\|\nabla \varphi\|_{L^{2}(\Omega)} \\
& \leq C \varepsilon\left\|u_{0}\right\|_{H^{2}(\Omega)}\|\nabla \varphi\|_{L^{2}(\Omega)},
\end{aligned}
$$

where we have used Proposition 2.2.

Then we rearrange equation (3.1), together with (3.3)-(3.5), to show that

$$
\begin{aligned}
& \left|\int_{\Omega}\left[A\left(x / \varepsilon, \nabla u_{\varepsilon}\right)-A\left(x / \varepsilon, \nabla v_{\varepsilon}\right)\right] \cdot \nabla \varphi d x+\int_{\Omega}\left(p_{0}-p_{\varepsilon}-\chi\right) \operatorname{div} \varphi d x\right| \\
& \quad \leq C \varepsilon\left\|u_{0}\right\|_{H^{2}(\Omega)}\|\nabla \varphi\|_{L^{2}(\Omega)} .
\end{aligned}
$$

Then let $\varphi=u_{\varepsilon}-v_{\varepsilon}=u_{\varepsilon}-u_{0}-\varepsilon N\left(x / \varepsilon, T_{\varepsilon}\left(\nabla \tilde{u}_{0}\right)\right)+\omega_{\varepsilon}$. By the coercive condition and the equation satisfied by $\omega_{\varepsilon}$, we can get the desired result, which completes the proof. 
Proof of Theorem 2 According to Theorem 1, we obtain the estimate

$$
\left\|u_{\varepsilon}-u_{0}-\varepsilon N\left(x / \varepsilon, T_{\varepsilon}\left(\nabla \tilde{u}_{0}\right)\right)+\omega_{\varepsilon}\right\|_{H_{0}^{1}(\Omega)} \leq C \varepsilon\left\|u_{0}\right\|_{H^{2}(\Omega)} .
$$

Hence, it suffices to show that

$$
\left\|\omega_{\varepsilon}\right\|_{H^{1}(\Omega)} \leq C \varepsilon^{1 / 2}\left\|u_{0}\right\|_{H^{2}(\Omega)} .
$$

In fact, by equation (3.1) and energy estimate, we obtain

$$
\begin{aligned}
\left\|\omega_{\varepsilon}\right\|_{H^{1}(\Omega)} & \\
\leq & C \varepsilon\left\|\operatorname{div} N\left(x / \varepsilon, T_{\varepsilon}\left(\nabla \widetilde{u}_{0}\right)\right)\right\|_{L^{2}(\Omega)}+C \varepsilon\left\|N\left(x / \varepsilon, T_{\varepsilon}\left(\nabla \widetilde{u}_{0}\right)\right)\right\|_{H^{1 / 2}(\partial \Omega)} \\
\leq & C \varepsilon\left\|\eta_{\varepsilon} N\left(x / \varepsilon, T_{\varepsilon}\left(\nabla \widetilde{u}_{0}\right)\right)\right\|_{H^{1}(\Omega)} \\
\leq & C \varepsilon\left\|\nabla_{\xi} N(y, \xi) T_{\varepsilon}\left(\nabla^{2} \widetilde{u}_{0}\right)\right\|_{L^{2}\left(\Omega_{\varepsilon}\right)}+C \| \nabla_{y} N\left(y, T_{\varepsilon}\left(\nabla \tilde{u}_{0}\right) \|_{L^{2}\left(\Omega_{\varepsilon}\right)}\right. \\
& +C \varepsilon\left\|\eta_{\varepsilon} N\left(x / \varepsilon, T_{\varepsilon}\left(\nabla \widetilde{u}_{0}\right)\right)\right\|_{L^{2}(\Omega)}+C\left\|N\left(x / \varepsilon, T_{\varepsilon}\left(\nabla \tilde{u}_{0}\right)\right)\right\|_{L^{2}\left(\Omega_{\varepsilon}\right)} \\
\leq & C \varepsilon\left\|T_{\varepsilon}\left(\nabla^{2} \widetilde{u}_{0}\right)\right\|_{L^{2}(\Omega)}+C\left\|T_{\varepsilon}\left(\nabla \widetilde{u}_{0}\right)\right\|_{L^{2}\left(\Omega_{\varepsilon}\right)}+C \varepsilon\left\|T_{\varepsilon}\left(\nabla \widetilde{u}_{0}\right)\right\|_{L^{2}(\Omega)} \\
\leq & C \varepsilon^{1 / 2}\left\|u_{0}\right\|_{H^{2}(\Omega)},
\end{aligned}
$$

where we have used the estimates in Proposition 2.2, Proposition 2.4, and Proposition 2.5.

This completes the proof of Theorem 2.

Proof of Theorem 3 It follows from Theorem 2 and Proposition 2.2, together with Minkowski's inequality, that

$$
\begin{aligned}
\left\|u_{\varepsilon}-u_{0}\right\|_{L^{2}(\Omega)} & \leq C \varepsilon^{1 / 2}\left\|u_{0}\right\|_{H^{2}(\Omega)}+\left\|\varepsilon N\left(x / \varepsilon, T_{\varepsilon}\left(\nabla \tilde{u}_{0}\right)\right)\right\|_{L^{2}(\Omega)} \\
& \leq C \varepsilon^{1 / 2}\left\|u_{0}\right\|_{H^{2}(\Omega)}+C \varepsilon\left\|T_{\varepsilon}\left(\nabla \tilde{u}_{0}\right)\right\|_{L^{2}\left(\mathbb{R}^{n}\right)} \\
& \leq C \varepsilon^{1 / 2}\left\|u_{0}\right\|_{H^{2}(\Omega)},
\end{aligned}
$$

which completes the proof of Theorem 3.

\section{Acknowledgements}

The authors would like to thank the reviewers for their valuable comments and helpful suggestions to improve the quality of this paper. The part of this work was done while the author was visiting School of Mathematics and Applied Statistics, University of Wollongong, Australia.

\section{Funding}

This work has been supported by the Natural Science Foundation of China (No. 11626239), China Scholarship Council (No. 201708410483), as well as Foundation of Education Department of Henan Province (No. 18A110037).

\section{Availability of data and materials}

Not applicable.

\section{Competing interests}

The authors declare that they have no competing interests.

Authors' contributions

All authors read and approved the manuscript. 


\section{Publisher's Note}

Springer Nature remains neutral with regard to jurisdictional claims in published maps and institutional affiliations.

Received: 17 March 2019 Accepted: 16 May 2019 Published online: 24 May 2019

\section{References}

1. Aleksanyan, H., Shahgholian, H., Sjölin, P.: Applications of Fourier analysis in homogenization of Dirichlet problem I. Pointwise estimates. J. Differ. Equ. 254, 2626-2637 (2013)

2. Aleksanyan, H., Shahgholian, H., Sjölin, P.: Applications of Fourier analysis in homogenization of the Dirichlet problem: $L^{p}$ estimates. Arch. Ration. Mech. Anal. 215, 65-87 (2015)

3. Alghamdi, A.M., Gala, S., Ragusa, M.A.: On the blow-up criterion for incompressible Stokes-MHD equations. Results Math. 73, 110 (2018)

4. Bensoussan, A., Lions, J.L., Papanicolaou, G.: Asymptotic Analysis for Periodic Structures. North-Holland, Amsterdam (1978)

5. Gala, S., Ragusa, M.A.: A new regularity criterion for the Navier Stokes equations in terms of the two components of the velocity. Electron. J. Qual. Theory Differ. Equ. 2016, 26 (2016)

6. Gérard, D., Masmoudi, N.: Homogenization and boundary layers. Acta Math. 209, 133-178 (2012)

7. Gu, S.: Convergence rates in homogenization of Stokes systems. J. Differ. Equ. 260, 5796-5815 (2016)

8. Gu, S., Shen, Z: Homogenization of Stokes systems and uniform regularity estimates. SIAM J. Math. Anal. 47 4025-4057 (2015)

9. Gu, S., Xu, Q:: Optimal boundary estimates for Stokes systems in homogenization theory. SIAM J. Math. Anal. 49 3831-3853 (2016)

10. Gu, S., Zhuge, J.: Periodic homogenization of Green's functions for Stokes systems (2017) arXiv:1710.05383v2

11. Huang, L., Lian, R.: Regularity to the spherically symmetric compressible Navier-Stokes equations with density-dependent viscosity. Bound. Value Probl. 2018, 85 (2018)

12. Jikov, V., Kozlov, S., Oleinik, O.: Homogenization of Differential Operators and Integral Functionals. Springer, Berlin (1994)

13. Kenig, C.E., Lin, F.H., Shen, Z.W.: Convergence rates in $L^{2}$ for elliptic homogenization problems. Arch. Ration. Mech. Anal. 203, 1009-1036 (2012)

14. Kenig, C.E., Lin, F.H., Shen, Z.W.: Periodic homogenization of Green and Neumann functions. Commun. Pure Appl. Math. 67, 1219-1262 (2012)

15. Pakhnin, M.A., Suslina, T.A.: Operator error estimates for the homogenization of the elliptic Dirichlet problem in a bounded domain. St. Petersburg Math. J. 24, 949-976 (2013)

16. Pastukhova, S.E.: Operator estimates in nonlinear problems of reiterated homogenization. Proc. Steklov Inst. Math. 261, 214-228 (2008)

17. Shen, Z.W.: Boundary estimates in elliptic homogenization. Mathematics 10,653-694 (2017)

18. Shen, Z.W., Zhuge, J.: Convergence rates in periodic homogenization of systems of elasticity. Proc. Am. Math. Soc. $145,1187-1202(2016)$

19. Shen, Z.W., Zhuge, J.: Boundary layers in periodic homogenization of Neumann problems. Commun. Pure Appl. Math. 71, 2163-2219 (2018)

20. Suslina, T.: Homogenization of the Dirichlet problem for elliptic systems: $L^{2}$-operator error estimates. Mathematika 59 463-476 (2013)

21. Suslina, T.: Homogenization of the Neumann problem for elliptic systems with periodic coefficients. SIAM J. Math Anal. 45, 3453-3493 (2013)

22. Wang, L., Xu, Q., Zhao, P.: Convergence rates on periodic homogenization of $\mathrm{p}$-Laplace type equations (2018) arXiv:1812.04837

23. Zhang, Z., Zhong, D., Cao, S., Qiu, S.: Fundamental Serrin type regularity criteria for 3D MHD fluid passing through the porous medium. Filomat 31, 1287-1293 (2017)

24. Zhao, J.: Homogenization of the boundary value for the Neumann problem. J. Math. Phys. 56, 021508 (2015)

\section{Submit your manuscript to a SpringerOpen ${ }^{\circ}$ journal and benefit from:}

- Convenient online submission

- Rigorous peer review

- Open access: articles freely available online

- High visibility within the field

- Retaining the copyright to your article

Submit your next manuscript at $\boldsymbol{~ s p r i n g e r o p e n . c o m ~}$ 